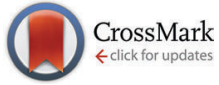

Cite this: J. Mater. Chem. C, 2017, 5, 1544

\title{
Stretchable heaters with composites of an intrinsically conductive polymer, reduced graphene oxide and an elastomer for wearable thermotherapy $\dagger$
}

\author{
Rui Zhou, Pengcheng Li, Zeng Fan, Donghe Du and Jianyong Ouyang*
}

\begin{abstract}
Thermal therapy is an effective physical treatment method for arthritis, stiff muscles, joint injuries, and injuries to the deep tissue of skin. Stretchable or even wearable electric heaters with uniform heating behavior are regarded as the next-generation electronic devices, which have been extensively studied for the personal thermal management and healthcare purpose. In this work, highly stretchable electrothermal heaters were developed by using composites of intrinsically conductive poly(3,4ethylenedioxythiophene):poly(styrene sulfonic acid) (PEDOT:PSS), elastomeric waterborne polyurethane (WPU) and reduced graphene oxide (rGO). rGO was mixed into the PEDOT:PSS/WPU blends to improve the temperature uniformity because $\mathrm{rGO}$ has high thermal conductivity while the polymers have very low thermal conductivity. The PEDOT:PSS/WPU/1 wt\% rGO composite film exhibits an electrical conductivity of $18.2 \mathrm{~S} \mathrm{~cm}^{-1}$ and an elongation at break of $530 \%$. The electrothermal performances of the polymer heaters were investigated with respect to the applied voltage, tensile strain, and the voltage on/off cycling process. The heater shows stable heating behavior under repetitive voltage on/off cycles, and the temperature remains almost unchanged under a tensile strain of up to $30 \%$. The devices can be comfortably attached to the skin of humans, for example on the wrist, and they exhibit a uniform and stable heating profile even under mechanical disturbance. Due to their outstanding stretchability, biocompatibility, desirable electrical and thermal conductivities, the WPU/PEDOT:PSS/rGO composites can be used in wearable and long-term thermotherapy applications.
\end{abstract}

Received 8th November 2016, Accepted 9th January 2017

DOI: $10.1039 / \mathrm{c} 6 \mathrm{tc0} 4849 \mathrm{~h}$

rsc.li/materials-c

\section{Introduction}

The prevalence of arthritis, stiff muscles, injuries to the deep tissue of the skin and joint injuries due to aging, obesity, or occupational overuse has been a big concern in healthcare. They are usually accompanied by symptoms like pain, stiffness, swelling and muscle weakness. ${ }^{1}$ Many treatment strategies have been developed to alleviate these symptoms. Among them thermotherapy is one of the most popular physical treatments. ${ }^{2,3}$ Thermotherapy involves the use of heat in the temperature range of 41 to $77^{\circ} \mathrm{C}$ for pain and stiffness relief. The most conventional heat therapy is in the form of heat packs. However, they suffer from problems of mechanical rigidity and heavy weight. In addition, the temperature is difficult to control and be tailored to the personal needs. As a consequence, the development of

Department of Materials Science \& Engineering, National University of Singapore,

7 Engineering Drive 1 117574, Singapore. E-mail: mseoj@nus.edu.sg,

pengchengli2013@gmail.com

$\dagger$ Electronic supplementary information (ESI) available: Raman spectra, XPS spectra, time-dependent temperature profiles, long time stability and relative resistance as a function of stretch cycles. See DOI: 10.1039/c6tc04849h flexible or even stretchable electrical heaters with controllable temperature and uniform heating behavior is of paramount importance for personal thermal management and healthcare purposes. Meanwhile, non-toxicity, together with stable electrical and thermal properties under the mechanical disturbance, is also required for a wearable heater.

Various efforts have been made to develop stretchable electric heaters by using metallic nanoparticles or nanowires, metal grids and carbon nanotubes. Examples include conductive fillers embedded in elastomers, ${ }^{4-7}$ or coated on stretchable fabrics, ${ }^{8-10}$ electrospun rubber fibers incorporated with silver nanoparticles, ${ }^{11}$ and interstitial regions of elastomers filled with liquid metal. ${ }^{12}$ However, the utilization of nanoparticles or nanowires may cause possible toxic effects, because these nanoparticles and nanowires are capable of entering the human body by inhalation, ingestion or skin penetration and they can interact with intracellular structures. ${ }^{12,13}$ In addition, the high cost of metallic nanowires and carbon nanotubes may also arouse economic concerns, making them unappealing for the large-area industrial applications. Therefore, intrinsically conductive polymers have received increasing research interest. The attractive properties 


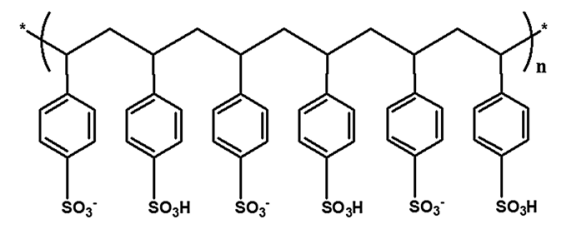

PSS
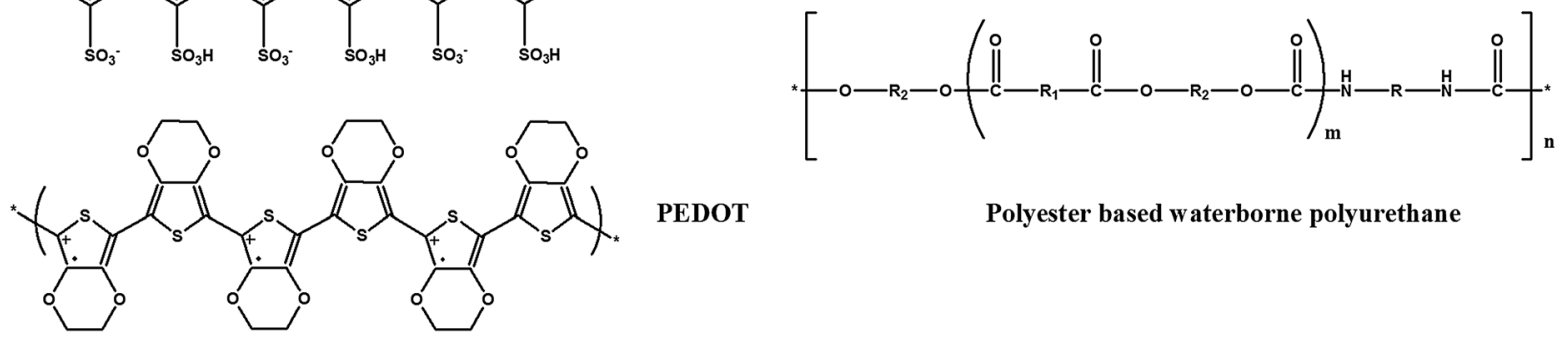

PEDOT

Polyester based waterborne polyurethane

Fig. 1 Chemical structures of PEDOT:PSS and polyester based WPU (R, R1 and R2 stand for different alkyl or aryl groups).

of conductive polymers, including their low cost, light weight, good processability and tunable electrical conductivity, make them excellent candidates for stretchable electric heaters. ${ }^{14-17}$ Water-dispersible poly(3,4-ethylenedioxythiophene):poly(styrene sulfonic acid) (PEDOT:PSS, chemical structure shown in Fig. 1) is regarded as one of the conductive polymers having potential for commercial and practical applications. This is due to its facile synthesis, high conductivity, good biocompatibility, and excellent transparency in the visible range. ${ }^{18-21}$ PEDOT:PSS films with high mechanical flexibility, and excellent thermal and electrical conductivity can be readily achieved through a convenient solution casting technique. ${ }^{22-24}$

In this work, highly stretchable composites of waterborne polyurethane (WPU, chemical structure shown in Fig. 1), PEDOT: PSS and reduced graphene oxide (rGO) (WPU/PEDOT:PSS/rGO) were prepared through the simple solution casting method, followed by the chemical reduction of GO in the WPU/PEDOT: PSS/GO composites using hydriodic acid (HI). rGO sheets with outstanding thermal conductivity (3000-5000 $\mathrm{W} \mathrm{m}^{-1} \mathrm{~K}^{-1}$ ) were incorporated into the polymer blends to improve heat conduction, and consequently, the temperature uniformity of the electric heater could be achieved. ${ }^{25,26}$ A reasonably high electrical conductivity of $18.2 \mathrm{~S} \mathrm{~cm}^{-1}$ and an extremely large elongation at break of 530\% were observed for the composite with the PEDOT:PSS loading of $5 \mathrm{wt} \%$ and rGO loading of $1 \mathrm{wt} \%$. The temperature of prestretched WPU/PEDOT:PSS/rGO films remains almost unchanged under the applied tensile strain of up to $30 \%$. The composite film shows stable switching behavior, and can be comfortably attached to human joints for effective thermotherapy treatments.

\section{Experimental section}

\subsection{Materials}

PEDOT:PSS aqueous solution (Clevios PH 1000 Lot 2014P0146) was obtained from Heraeus. It had a concentration of $1.3 \mathrm{wt} \%$ by weight and a PSS-to-PEDOT weight ratio of 2.5. Water-borne polyurethane (WPU-3-505G) was supplied by the Taiwan PU Corporation, which is a polyester-based nonionic polyurethane water dispersion with a concentration of $39.8 \mathrm{wt} \%$. Dimethyl sulfoxide (DMSO) and HI (Sigma-Aldrich, $57 \mathrm{wt} \%$ in water) solution were obtained from Sigma-Aldrich. Highly concentrated
GO dispersion in water (5 $\mathrm{mg} \mathrm{mL}^{-1}$ ) was purchased from Graphene Supermarket. All the chemicals were used as received without further purification.

\subsection{Preparation of polymer films}

PEDOT:PSS solution was added to $5 \mathrm{wt} \%$ DMSO, and it was then mixed with $20 \mathrm{wt} \%$ water-borne polyurethane solution by vigorously stirring at room temperature for half an hour. The weight ratio of PEDOT:PSS to WPU was 1/19. The PEDOT:PSS/ WPU blend films were prepared by drop casting the dispersions in a glass Petri dish. They were then dried at $70{ }^{\circ} \mathrm{C}$ in an oven for $12 \mathrm{~h}$. The polymer blend films with a thickness of $0.1 \mathrm{~mm}$ were used for mechanical and electrical tests.

WPU/PEDOT:PSS/rGO composite films were prepared by adding graphene oxide (GO) into WPU/PEDOT:PSS aqueous solutions. The loadings of GO and PEDOT:PSS are $1 \mathrm{wt} \%$ and $5 \mathrm{wt} \%$, respectively, with respect to the total weight of PEDOT: PSS, WPU and GO. GO was converted to rGO by immersing the composite film into a $\mathrm{HI}$ (Sigma-Aldrich, $57 \mathrm{wt} \%$ in water) solution at room temperature for $2 \mathrm{~min}$. The PEDOT:PSS/WPU/ rGO composite films were washed with ethanol and deionized water several times. They were then dried at $70{ }^{\circ} \mathrm{C}$ overnight.

\subsection{Polymer film heaters}

Two $\mathrm{Cu}$ wires were connected to the two ends of a free-standing film of $10 \mathrm{~mm} \times 20 \mathrm{~mm}$ using silver paste. The temperature of the film was increased by applying a DC voltage to the two $\mathrm{Cu}$ wires. The temperatures of the film in the middle and side positions were measured using a thermocouple. The temperature distribution of the film was measured using a VarioCAM high resolution infrared camera, which was mounted on the top surface of the film.

\subsection{Characterization}

Raman spectra were recorded using a Horiba Scientific LabRAM HR Evolution system with a $514 \mathrm{~nm} \mathrm{Ar}+$ laser as the excitation source. Scanning electron microscopy (SEM) images were obtained using a Zeiss Supra 40 field emission scanning electron microscope. X-ray photoelectron spectroscopy (XPS) measurements were performed using an Axis Ultra DLD X-ray photoelectron spectrometer equipped with an $\mathrm{Al} \mathrm{K} \alpha$ X-ray source 
(1486.6 eV photons). The electrical conductivities of polymer films were measured by the four-point probe technique with four copper wires in parallel and a Keithley 2400 source meter. The four wires were connected to a polymer film with silver paste. The tensile tests were performed using an Instron tester 5567 equipped with a $100 \mathrm{~N}$ load cell at a crosshead speed of $20 \mathrm{~mm} \mathrm{~min}^{-1}$. Rectangle strips with dimensions of $20 \times 2 \times$ $0.1 \mathrm{~mm}^{3}$ were used for the mechanical tests. The changes in the relative resistance $\left(R / R_{0}\right)$ under tensile strain were collected using a Keithley 2400 source/meter combined with the Instron tester. Two $\mathrm{Cu}$ wires were connected to the two ends of a rectangular composite film $(10 \mathrm{~mm} \times 20 \mathrm{~mm})$ using silver paste. The copper wires were connected to a Keithley 2400 source meter to measure 2-point resistance during the stretching test.

\section{Results and discussion}

\subsection{Structure and morphology of polymer composite films}

The WPU/PEDOT:PSS/rGO composite films with $5 \mathrm{wt} \%$ PEDOT: PSS and $1 \mathrm{wt} \%$ rGO were used as the stretchable polymer heaters in this study. The composite film was prepared by blending GO into PEDOT:PSS/WPU and then reduced GO into rGO by immersing the composite film into a HI solution. The characterization of GO has been reported by our early works. ${ }^{27-29}$ The GO has a size distribution from 40 to $900 \mathrm{~nm}$ and the single layer structure as revealed by TEM and dynamic light scattering (DLS) (Fig. S1, ESI $\dagger$ ). The reduction of GO by HI was confirmed by Raman spectroscopy (Fig. S2 in the ESI $\dagger$ ). Previous studies have demonstrated that hydriodic acid (HI) is an effective agent to reduce GO into rGO. ${ }^{28,30,31}$ As shown in Fig. S2 (ESI $\dagger$ ), both WPU/PEDOT:PSS/GO and WPU/PEDOT:PSS/rGO composite films display prominent Raman bands at 1350 and $1600 \mathrm{~cm}^{-1}$, which are assigned to the D and $\mathrm{G}$ bands of GO or rGO, respectively. The $\mathrm{D}$ band is attributed to the disorders or defects in the graphene sheets, while the $G$ band originates from the $E_{2 g}$ phonon of $\mathrm{sp}^{2}$ carbon atoms. ${ }^{32}$ The peak position of the $\mathrm{G}$ band appears at $1604 \mathrm{~cm}^{-1}$ for the WPU/PEDOT:PSS/GO composite film, and it shifts to $1595 \mathrm{~cm}^{-1}$ after the reduction of GO by $\mathrm{HI}$. The relative intensity ratio of the $\mathrm{D}$ band to the $\mathrm{G}$ band $\left(I_{\mathrm{D}} / I_{\mathrm{G}}\right)$ changes remarkably after the reduction. It can be used to measure the defect density of graphene-based materials. ${ }^{32-34}$ The value of the $I_{\mathrm{D}} / I_{\mathrm{G}}$ ratio increases from 1.15 for the pristine WPU/PEDOT:PSS/GO film to 1.29 after HI treatment. The Raman spectrum of the crosssection of the WPU/PEDOT:PSS/GO film after the HI treatment shows a similar $I_{\mathrm{D}} / I_{\mathrm{G}}$ ratio to that of the sample surface. These results demonstrate that the $\mathrm{HI}$ treatment introduces more structural defects into the carbon basal plane, and thus confirms the successful reduction of GO by $\mathrm{HI}$.

Fig. 2 presents the SEM images of polymer blend and composite films. It can be seen that the polymer blend films exhibit a uniform surface morphology, and no obvious phase segregation can be observed. This suggests that PEDOT:PSS is miscible with WPU. ${ }^{35}$ This can be attributed to the interactions between the PSS component of PEDOT:PSS and the WPU polymer chains. The oxygen atoms of PSS can form hydrogen
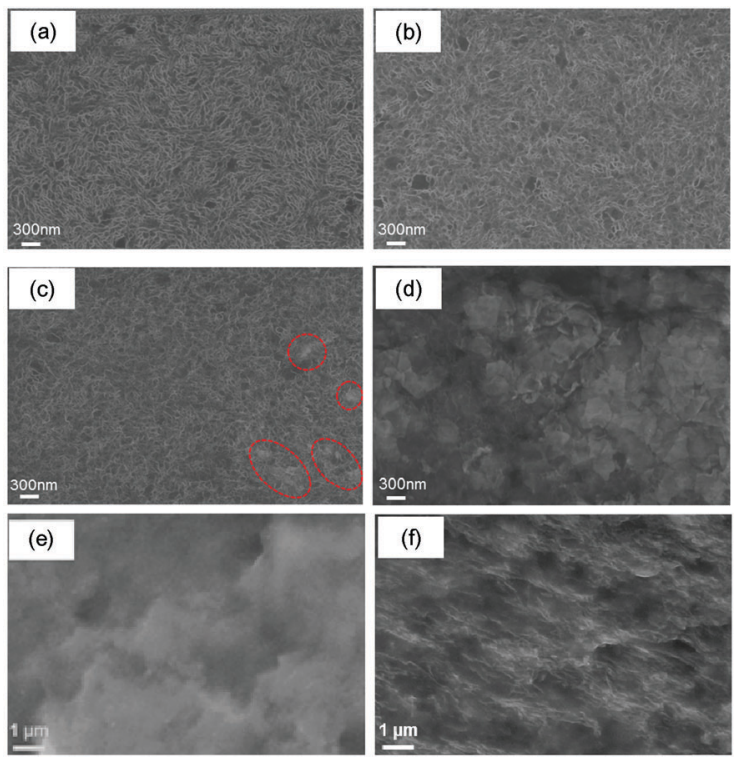

Fig. 2 SEM images of surface of (a) a WPU/PEDOT:PSS blend film; (b) a HI treated WPU/PEDOT:PSS blend film; (c) a WPU/PEDOT:PSS/1 wt\% rGO composite film; (d) a WPU/PEDOT:PSS/5 wt\% rGO composite film and cross-section of (e) a WPU/PEDOT:PSS blend film; (f) a WPU/PEDOT:PSS/1 wt\% rGO composite film.

bonds with the $\mathrm{N}-\mathrm{H}$ groups of WPU. In addition, protons can transfer from PSSH to WPU, giving rise to the negatively charged $-\mathrm{SO}_{3}{ }^{-}$groups of PSS and positively charged $-\mathrm{NH}_{2}{ }^{+}$ groups of WPU and thus Coulombic interactions between them. Compared with the as-prepared WPU/PEDOT:PSS film (Fig. 2a), the surface seems to become rougher for the HI-treated WPU/PEDOT:PSS film (Fig. 2b). It can be due to the loss of some PSS polymer chains occurring during the acid treatment as well as the ethanol and deionized water washing process. When the composite film is immersed in the HI acid solution, HI may penetrate into the film, the anions of acid may replace some PSS as the counter ions for PEDOT, and the protons can be transferred to the PSS $^{-}$chains and it leads to the formation of neutral PSSH. ${ }^{34,35}$ As a result, PSSH can be washed away with water because of the absence of Coulombic attractions between PEDOT and PSSH. The presence of rGO nanosheets can be clearly identified in the SEM images of WPU/PEDOT:PSS/rGO composite films. At an rGO content of $1 \mathrm{wt} \%$, individual nanosheets are well dispersed in the polymer matrix (Fig. 2c). It is attributed to the good compatibility between hydrophilic GO with WPU and PEDOT:PSS. However, severe aggregation of nanosheets occurs at a rGO content of $5 \mathrm{wt} \%$ (Fig. 2d), which may significantly deteriorate the mechanical elasticity of the film as to be discussed later. The dispersion of rGO in the WPU/ PEDOT:PSS/1 wt\% rGO composite film was further confirmed by the cross-section SEM images of the WPU/PEDOT:PSS blend film and the WPU/PEDOT:PSS/1 wt\% rGO composite film (Fig. 2e and f). RGO nanosheets are uniformly dispersed in the PEDOT:PSS/WPU matrix without any large aggregation.

The loss of some PSS polymer chains after acid treatment was further verified by XPS spectra. Fig. S3 in the ESI, $\dagger$ shows 
Table 1 Electrical conductivity and tensile properties of the as-prepared WPU/PEDOT:PSS, HI-treated WPU/PEDOT:PSS, and WPU/PEDOT:PSS/ rGO composite films

\begin{tabular}{llll}
\hline & $\begin{array}{l}\text { Electrical } \\
\text { conductivity } \\
\left(\mathrm{S} \mathrm{cm}^{-1}\right)\end{array}$ & \begin{tabular}{l} 
Tensile property \\
\cline { 4 - 4 } Poung's \\
modulus $(\mathrm{Mpa})$
\end{tabular} & $\begin{array}{l}\text { Strain at } \\
\text { break }(\%)\end{array}$ \\
\hline $\begin{array}{l}\text { As-prepared WPU/ } \\
\text { PEDOT:PSS }\end{array}$ & $12.5 \pm 0.4$ & $18.3 \pm 0.7$ & 860 \\
$\begin{array}{l}\text { HI-treated WPU/ } \\
\text { PEDOT:PSS }\end{array}$ & $18.1 \pm 0.3$ & $34.3 \pm 0.6$ & 600 \\
$\begin{array}{l}\text { WPU/PEDOT:PSS/ } \\
\begin{array}{l}\text { 1 wt\% rGO } \\
\text { WPU/PEDOT:PSS/ }\end{array}\end{array}$ & $18.2 \pm 0.2$ & $246.2 \pm 8.6$ & 530 \\
5 wt\% rGO & $14.0 \pm 0.2$ & $357.0 \pm 15.8$ & 13 \\
\end{tabular}

the S2p XPS spectra of the WPU/PEDOT:PSS blend film before and after $\mathrm{HI}$ treatment. The two bands with binding energies between 166 and $172 \mathrm{eV}$ are attributed to the sulphur atoms of PSS, while the bands between 162 and $166 \mathrm{eV}$ originate from the sulfur atoms of PEDOT. ${ }^{36}$ It is clear that the relative intensity ratio of PEDOT to PSS increases after acid treatment, suggesting the removal of some PSS in the blend film. As PSS is the counter ion for PEDOT in the composite film, the removal of some excess PSS leads to the increase in the conductivity of the composite film. ${ }^{37}$

\subsection{Electrical and mechanical properties of polymer films}

Conductivity is one of the most important parameters for the electronic systems, which can greatly affect the performance of the electronic devices. As the pristine PEDOT:PSS film has a low conductivity below $1 \mathrm{~S} \mathrm{~cm}^{-1}$, the addition of a polar solvent is an efficient way to increase the electrical conductivity of PEDOT:PSS. ${ }^{37}$ DMSO was used in this work to enhance the electrical conductivity of PEDOT:PSS. As shown in Table 1, the as-prepared WPU/PEDOT:PSS blend films have an electrical conductivity of $12.5 \mathrm{~S} \mathrm{~cm}^{-1}$, and the value increases to $18.1 \mathrm{~S} \mathrm{~cm}^{-1}$ after the $\mathrm{HI}$ treatment. This is due to the segregation of the insulating PSSH component from the blend film and the conformational change of the PEDOT chains after acid treatment. ${ }^{38-40}$ The addition of $1 \mathrm{wt} \%$ rGO hardly affects the conductivity of the polymer blends. This can be explained by the very low content of rGO fillers as well as their relatively low conductivity compared with the DMSO-added and HI-treated PEDOT:PSS. But the conductivity decreases to $14.0 \mathrm{~S} \mathrm{~cm}^{-1}$ when the rGO loading content increases to $5 \mathrm{wt} \%$.

The mechanical properties of the blend and composite films were investigated through a tensile test. Fig. 3 shows the representative stress-strain curves of a pristine WPU/PEDOT:PSS film, the HI-treated WPU/PEDOT:PSS film and WPU/PEDOT:PSS/rGO composite films. The variations of the Young's modulus and elongation at break are summarized in Table 1. The pristine WPU/PEDOT:PSS blend film shows a relatively low Young's modulus of $18.3 \mathrm{MPa}$, and a large elongation at break of $860 \%$. After the HI treatment, Young's modulus slightly increases, whereas the elongation at break decreases. This is ascribed to the removal of some PSSH chain from the WPU/PEDOT:PSS film. PSSH chains are more flexible than PEDOT because the former has a saturated main chain while the latter has a rigid

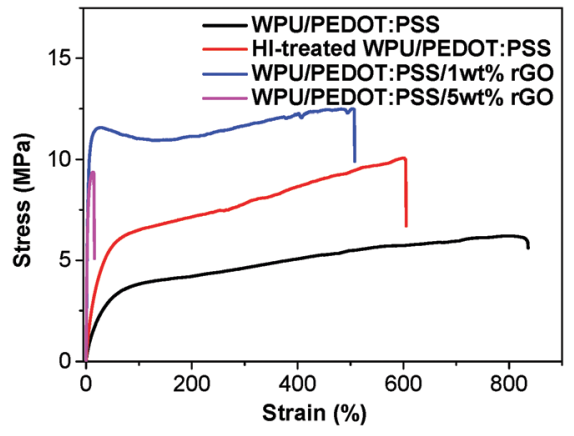

Fig. 3 Representative stress-strain curves of a pristine WPU/PEDOT:PSS film, HI-treated WPU/PEDOT:PSS film, and WPU/PEDOT:PSS/rGO composite films with $1 \mathrm{wt} \%$ and $5 \mathrm{wt} \% \mathrm{rGO}$

conjugated backbone. The incorporation of rGO into the polymer blend increases its Young's modulus while decreasing its flexibility because of the two-dimensional rigid conjugated structure of rGO. At $1 \mathrm{wt} \% \mathrm{rGO}$, the Young's modulus increases to $246.2 \mathrm{MPa}$, corresponding to an increase of $618 \%$. This significant increase by rGO can be attributed to its ultrahigh Young's modulus (about $1 \mathrm{TPa}),{ }^{41}$ and large specific surface area (about $2630 \mathrm{~m}^{2} \mathrm{~g}^{-1}$ ), ${ }^{42}$ which maximize the interaction area between the polymer phase and rGO. The elongation at break decreases slightly for the composite with $1 \mathrm{wt} \%$ rGO loading. It is presumably due to the interactions between rGO and the polymer matrix, which hinders the movement of polymer chains and make the material more brittle. The elongation at break decreases remarkably at $5 \mathrm{wt} \% \mathrm{rGO}$. Because the composite with a rGO loading of $1 \mathrm{wt} \%$ has better mechanical stretchability and electrical conductivity, it is chosen as the stretchable electric heater in this study.

\subsection{Electric heating behavior of polymer films}

The performance parameters of electrical heaters, including the steady heating temperature, temperature uniformity, cycling stability, and heating behaviour under mechanical disturbance, can dramatically influence their practical applications. These properties of the composite films were studied in detail to demonstrate their potential as electric heaters. The electric heating behaviors of the WPU/PEDOT:PSS film and the WPU/PEDOT:PSS/rGO composite film were investigated. A constant DC bias voltage was applied at the two ends of a rectangle film to induce electrically driven resistive Joule heating. Fig. $4 a-d$ show the time-dependent temperature profiles of the middle and side positions of the films (illustrated in Fig. $4 \mathrm{~g}$ ). The steadystate temperature increases synchronously with the increase of the applied voltage, indicating the temperature controllability of the electric heaters. Regardless of the different applied voltages, the temperature can be increased from room temperature to the equilibrium state within $3 \mathrm{~min}$. It is attributed to the relatively high conductivity of the composite films. The heating and cooling rates of the specimens are obtained from the derivative of the temperature with respect to time, and are plotted in Fig. $4 \mathrm{e}$ and $\mathrm{f}$. The maximum heating and cooling rate of the pristine WPU/PEDOT:PSS film under an applied voltage of $5 \mathrm{~V}$ is 

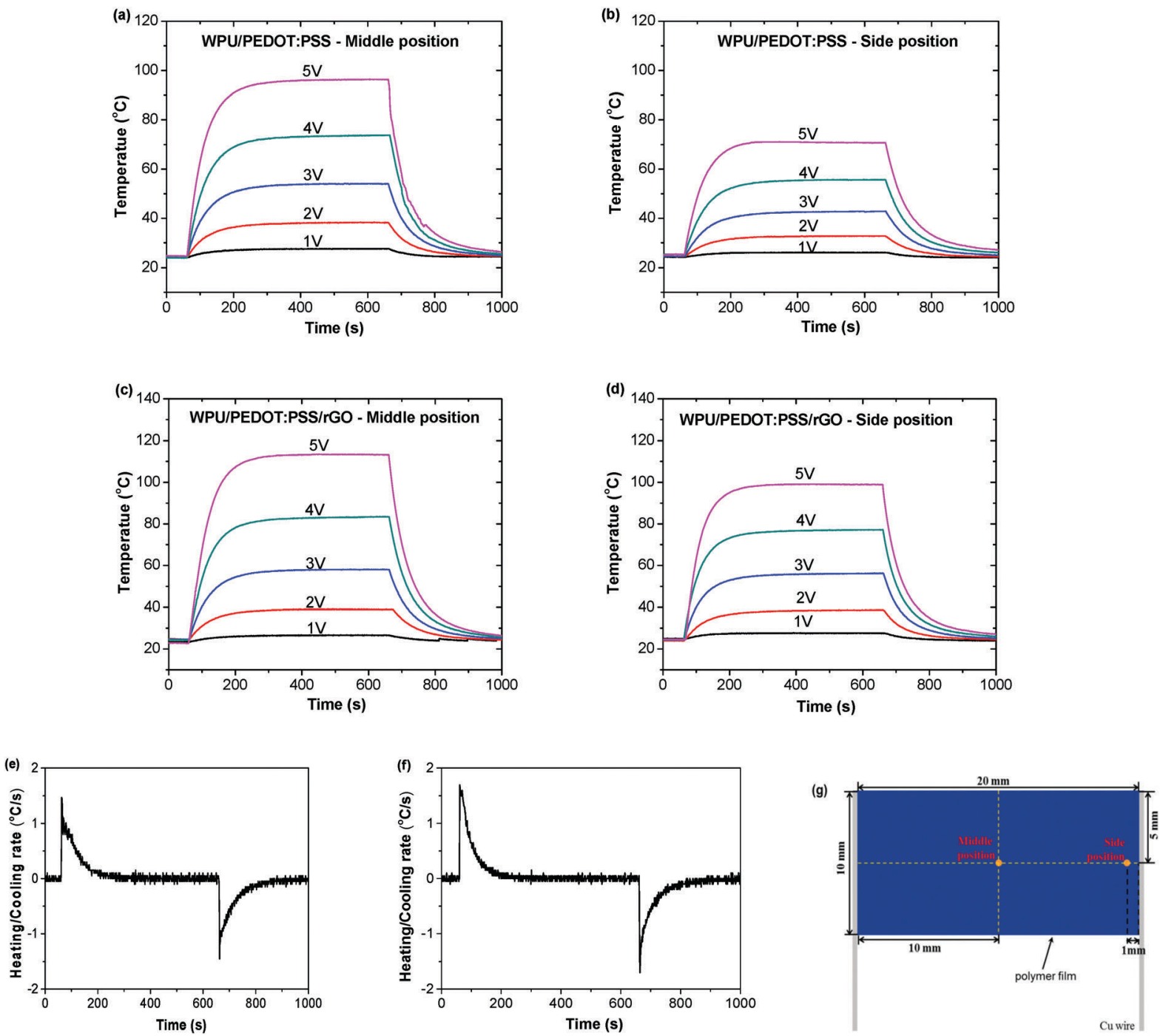

Fig. 4 Time-dependent temperature profiles of a WPU/PEDOT:PSS blend film in the (a) middle and (b) side position; time-dependent temperature profiles of a WPU/PEDOT:PSS/1 wt\% rGO composite film in the (c) middle and (d) side positions. The voltage applied to each sample was 1-5 V for (a-d); the variation of the temperature derivative with the time at the applied voltage of $5 \mathrm{~V}$ for a (e) WPU/PEDOT:PSS film and (f) WPU/PEDOT:PSS/1 wt\% rGO film; (g) schematic illustration of the polymer film heater.

about $\pm 1.47{ }^{\circ} \mathrm{C} \mathrm{s}^{-1}$, whereas it increases to $\pm 1.70{ }^{\circ} \mathrm{C} \mathrm{s}^{-1}$ for the WPU/PEDOT:PSS $/ 1$ wt $\%$ rGO film. This can be ascribed to the higher electrical conductivity and thermal conductivity of WPU/PEDOT:PSS/1 wt\% rGO than WPU/PEDOT:PSS.

In addition, it is expected that the incorporation of rGO can lead to a more uniform heat distribution. This is verified by measuring the temperature of the middle and side positions of the rectangle films using a thermocouple. For both specimens, this temperature difference becomes larger at higher voltages. It is presumably due to the faster heat conduction to the substrate as well as the heat convection to the surrounding air at higher temperatures. However, at a certain voltage, the temperature difference is much smaller for the rGO incorporated film in comparison with the WPU/PEDOT:PSS blend film. For example, under a voltage of $3 \mathrm{~V}$, the WPU/PEDOT:PSS film exhibits a middle-to-side temperature difference of $11.3{ }^{\circ} \mathrm{C}$ and a middle position temperature of $54{ }^{\circ} \mathrm{C}$. By contrast, the difference is only about $1.9{ }^{\circ} \mathrm{C}$, and the middle position temperature is $58{ }^{\circ} \mathrm{C}$ in the presence of the rGO fillers at the same applied voltage (Fig. 4c and d). To further confirm the improvement in the temperature uniformity arising from the presence of rGO, the temperature difference between the side and middle positions of a HI-treated PEDOT:PSS/WPU film was also measured (Fig. S4, $\mathrm{ESI}+$ ). The maximum heating rate at $5 \mathrm{~V}$ and the cooling rate of the HI-treated PEDOT:PSS/WPU film $\left( \pm 1.75{ }^{\circ} \mathrm{C} \mathrm{s}^{-1}\right)$ is almost the same as that of WPU/PEDOT:PSS/1 wt\% rGO film $\left( \pm 1.70{ }^{\circ} \mathrm{C} \mathrm{s}^{-1}\right)$. Both of them are higher than the as-prepared WPU/PEDOT:PSS $\left( \pm 1.47{ }^{\circ} \mathrm{C} \mathrm{s}^{-1}\right)$. This can be attributed to the higher electric conductivity of two former samples than the latter. The heating performance is similar for the $5 \mathrm{wt} \%$ PEDOT:PSS/WPU films with or without adding DMSO (Fig. S5, ESI $\dagger$ ). But the temperature uniformity is mainly related to the presence of rGO. At similar middle temperature, the temperature uniformity of the HI-treated PEDOT:PSS/WPU film is similar to that of the as-prepared 
PEDOT:PSS/WPU film. Both of them have less temperature uniformity than the WPU/PEDOT:PSS/ $1 \mathrm{wt} \%$ rGO film. These results indicate that the incorporation of a small amount of rGO can make the temperature more homogeneous. The above results demonstrate that rGO can function as an efficient thermal conduction promoter and help achieve a uniform heat distribution across the film.

The effect of the rGO fillers on the temperature distribution of the films was further investigated through IR imaging. Fig. 5 shows the temperature changes of WPU/PEDOT:PSS and WPU/PEDOT:PSS/rGO rectangle films along the length direction, and the insets show the corresponding IR images at different applied voltages. The film exhibits the highest temperature in the centre, and the temperature gradually decreases along the centre to the end of the film. For both WPU/PEDOT:PSS and WPU/PEDOT:PSS/1 wt\% rGO films, the maximum temperature difference becomes larger under a higher applied voltage, which is consistent with the results obtained using the thermocouple. In addition, at a voltage of $3 \mathrm{~V}$, the maximum temperature difference is about $10{ }^{\circ} \mathrm{C}$ for the WPU/PEDOT:PSS blend film, and decreases to $5{ }^{\circ} \mathrm{C}$ with the addition of rGO.

The cyclic electric heating and cooling tests were conducted to investigate the stability of the WPU/PEDOT:PSS/1 wt $\%$ rGO composite film as the electric heater. Fig. 6a shows the temperature on/off responses of a composite film under a voltage of $3 \mathrm{~V}$ (Fig. S6, $\mathrm{ESI} \dagger$ ). A constant DC voltage was applied to the film for $10 \mathrm{~min}$. The voltage was then shut off, and the sample was cooled down in air for $10 \mathrm{~min}$. The composite film can be readily switched between $60{ }^{\circ} \mathrm{C}$ and room temperature with stable electric heating behavior,
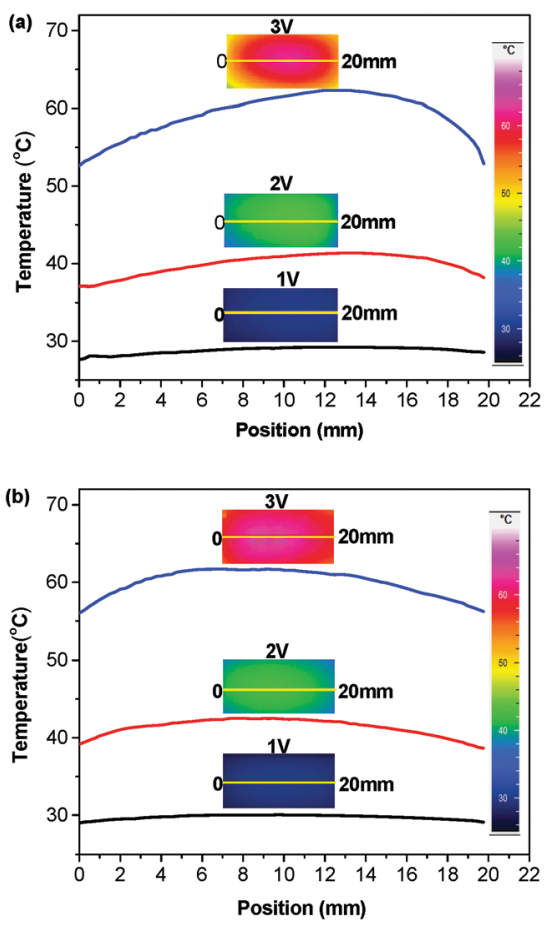

Fig. 5 Position-dependent temperature profiles of a (a) WPU/PEDOT:PSS and (b) WPU/PEDOT:PSS/1 wt\% rGO rectangle film along the length direction (as shown in the yellow line) at the applied voltage of $1-3 \mathrm{~V}$. The insets are the IR images at the three different voltages. and the heating and cooling processes have good repeatability. The WPU/PEDOT:PSS/1 wt\% rGO composite film as an electric heater also is quite stable in different moisture environments. As shown in Fig. 6b, the WPU/PEDOT:PSS/1 wt\% rGO film exhibits almost the same time-dependent temperature profiles under different humidity values when a $3 \mathrm{~V}$ voltage is applied to the film at $60 \mathrm{~s}$ and is stopped at $660 \mathrm{~s}$.

The elasticity of the WPU/PEDOT:PSS/1 wt\% rGO composite film was investigated. A $30 \%$ cyclic strain is applied to the film. Fig. $6 \mathrm{c}$ shows the tensile stress changes as tensile cycles. The maximum stress decreased in the first ten cycles and then tended to become stable with further stretching cycles. As can be seen from the cyclic strain-stress curves (Fig. 6d), the composite film could recover to $25.9 \%$ strain after the release of the stress in the first cycle, which is attributed to the plastic properties of WPU. In addition, the composite film can recover to $18.3 \%$ strain after 100 cycles.

As for the wearable articular thermotherapy applications, the devices should withstand the deformation of joints. An ideal electric heater should be insensitive to the strain in the range of $0-20 \%$. The performance of the WPU/PEDOT:PSS/1 wt $\%$ rGO composite film is evaluated by applying uniaxial tensile strain to the heater under a constant voltage. Fig. 6e shows the variation of the relative resistance $\left(R / R_{0}\right)$ with the tensile strain. The relative resistance increases to 1.05 after stretching to $20 \%$ strain for the WPU/PEDOT:PSS film without prestretching. The resistance of the composite film becomes less sensitive to the strain after it is prestretched to $30 \%$ and released. Its relative resistance remains almost unchanged in the strain range of $0-20 \%$. Even after the film was stretched and released for 200 cycles the relative resistance did not increase obviously (Fig. S7, ESI $\dagger$ ). The prestretching effect is attributed to the generation of minor cracks or phase separation within the composite film during the prestretching process, which increases the resistance at $0 \%$ strain and makes the film less sensitive to the strain thereafter. In addition, the maximum temperature measured in the strain range of $0-30 \%$ is also indicated in Fig. 6f. For the WPU/PEDOT:PSS/1 wt $\%$ rGO composite film without prestretching, the heater displays lower temperature when the strain is applied. It can be seen that the temperature decreases from $60{ }^{\circ} \mathrm{C}$ at $0 \%$ strain to $54{ }^{\circ} \mathrm{C}$ under the tensile strain of $30 \%$. Presumably, at a constant voltage heat generation by Joule-heating is inversely proportional to the resistance of the heaters, and the resistance of the composite film increases with increasing tensile strain. For the prestretched film, the initial temperature at $0 \%$ strain decreases to $56{ }^{\circ} \mathrm{C}$ because of the increased resistance induced by the prestretching process. The temperature remains almost constant within the strain of $0-30 \%$. Hence, the prestretched WPU/PEDOT:PSS/1 wt $\%$ rGO composite film can be used as a wearable articular thermotherapy device.

Moreover as both WPU and PEDOT:PSS are biocompatible materials, ${ }^{19-21,43}$ it is safe to use WPU/PEDOT:PSS/rGO composites for wearable electric heaters. A heater made of WPU/PEDOT:PSS/ ( $1 \mathrm{wt} \% \mathrm{rGO}$ ) was directly attached to the human wrist to study its performance under mechanical disturbances. Fig. 7 shows the 

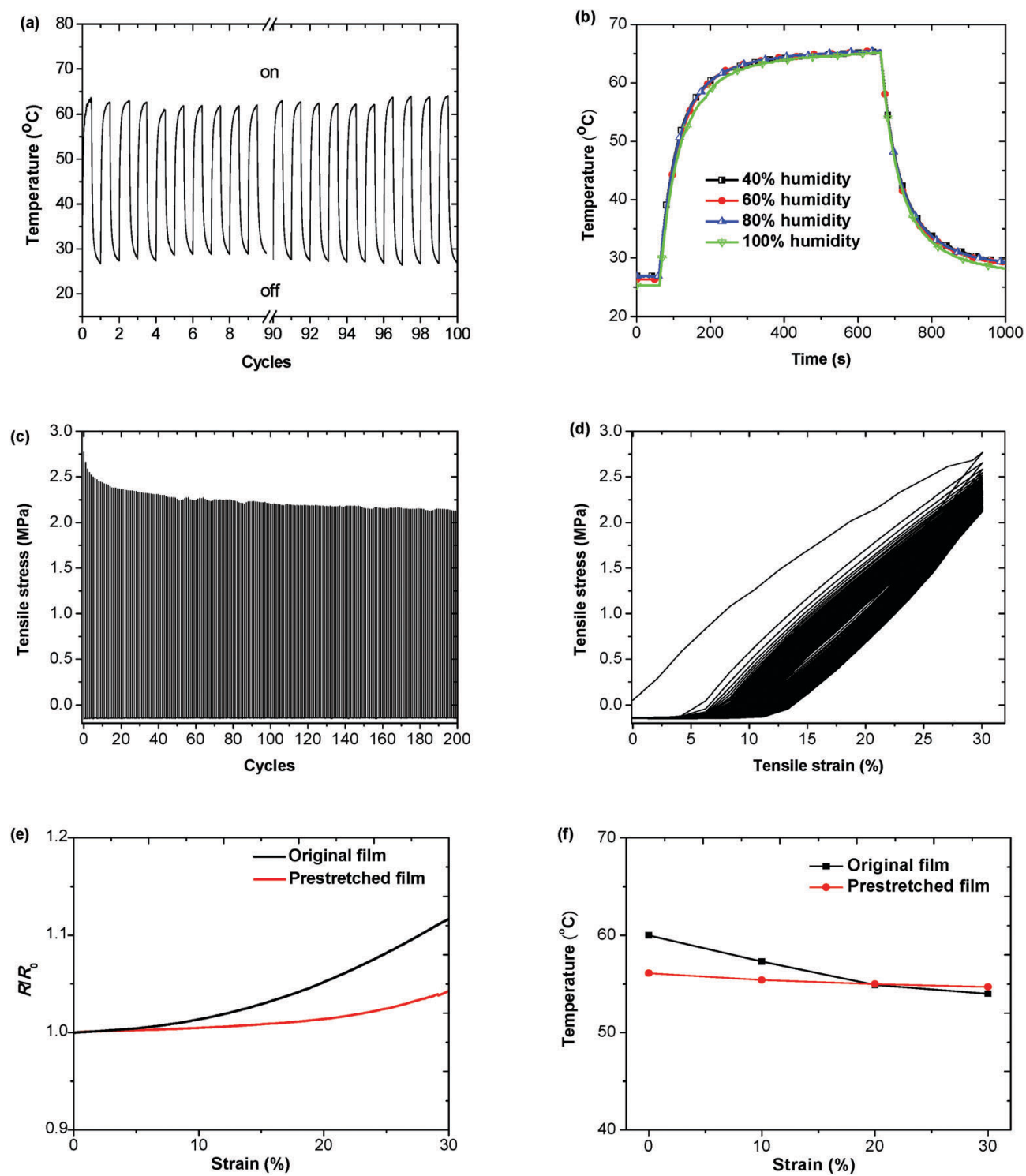

Fig. 6 (a) Temperature on/off responses of a WPU/PEDOT:PSS/1 wt\% rGO composite film under a voltage of 3 V; (b) time-dependent temperature profiles of a WPU/PEDOT:PSS/1 wt\% rGO composite film under different humidity; (c) tensile stress change as stretching cycles of WPU/PEDOT:PSS/ $1 \mathrm{wt} \%$ rGO composite film during the 30\% cyclic strain; (d) stress-strain curves of WPU/PEDOT:PSS $/ 1$ wt\% rGO composite film during the 30\% cyclic strain; variations of (e) the relative resistance $\left(R / R_{0}\right)$ and (f) the maximum temperature measured in the strain range of 0-30\% for the WPU/PEDOT:PSS/1 wt\% $\mathrm{rGO}$ films with and without prestretching. A voltage of $3 \mathrm{~V}$ was applied for each sample.
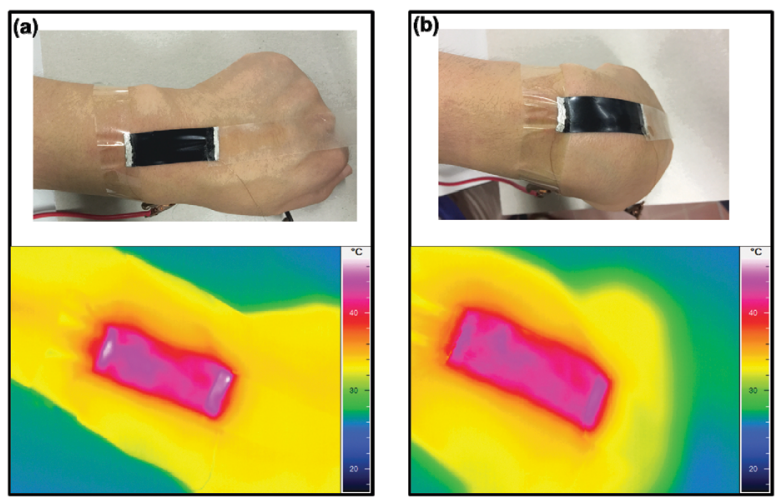

Fig. 7 Photograph and IR images of a WPU/PEDOT:PSS/1 wt\% rGO electric heater affixed to a human wrist at (a) neutral and (b) inward bending conditions. representative photograph and the IR image of the heater under neutral, and inward bending conditions. As can be observed, the WPU/PEDOT:PSS/1 wt\% rGO film maintains conformal contacts with the joint during general human motions, and the heater displays uniform heat distribution. It is attributed to its high electrical and thermal conductivity as well as good mechanical properties.

\section{Conclusion}

Highly stretchable and conductive WPU/PEDOT:PSS/rGO composite films were prepared through the facile solution casting technique, followed by $\mathrm{HI}$ acid treatment. The composite films can have an 
electrical conductivity of $18.2 \mathrm{~S} \mathrm{~cm}^{-1}$ and a strain at break of $530 \%$. The incorporation of $1 \mathrm{wt} \%$ rGO has negligible effects on the electrical conductivity but it significantly improves the thermal conduction of the films. It can then lead to more uniform temperature distributions for the heaters. Furthermore, the composite heaters show stable heating property under the repetitive voltage on/off cycles. The temperature remains almost unchanged under the tensile strain of $0-30 \%$. The heating profile on the wrist is uniform and stable despite joint movements. Therefore, the WPU/PEDOT:PSS/rGO composites are suitable for wearable heaters that can perform articular thermotherapy.

\section{Acknowledgements}

This work is financially supported by a research fund from the Ministry of Education, Singapore (R-284-000-136-112).

\section{Notes and references}

1 P. Sarzi-Puttini, M. A. Cimmino, R. Scarpa, R. Caporali, F. Parazzini, A. Zaninelli, F. Atzeni and B. Canesi, Semin. Arthritis Rheum., 2005, 35, 1.

2 S. Michlovitz, L. Hun, G. N. Erasala, D. A. Hengehold and K. W. Weingand, Arch. Phys. Med. Rehabil., 2004, 85, 1409.

3 J. Nakano, C. Yamabayashi, A. Scott and W. D. Reid, Phys. Ther. Sport, 2012, 13, 180.

4 S. Hong, H. Lee, J. Lee, J. Kwon, S. Han, Y. D. Suh, H. Cho, J. Shin, J. Yeo and S. H. Ko, Adv. Mater., 2015, 27, 4744.

5 S. Ding, J. Jiu, Y. Gao, Y. Tian, T. Araki, T. Sugahara, S. Nagao, M. Nogi, H. Koga, K. Suganuma and H. Uchida, ACS Appl. Mater. Interfaces, 2016, 8, 6190.

6 J. Yan and Y. G. Jeong, Mater. Des., 2015, 86, 72.

7 B. W. An, E.-J. Gwak, K. Kim, Y.-C. Kim, J. Jang, J.-Y. Kim and J.-U. Park, Nano Lett., 2016, 16, 471.

8 Y. Atwa, N. Maheshwari and I. A. Goldthorpe, J. Mater. Chem. C, 2015, 3, 3908.

9 P. Ilanchezhiyan, A. S. Zakirov, G. M. Kumar, S. U. Yuldashev, H. D. Cho, T. W. Kang and A. T. Mamadalimov, RSC Adv., 2015, 5, 10697.

10 P.-C. Hsu, X. Liu, C. Liu, X. Xie, H. R. Lee, A. J. Welch, T. Zhao and Y. Cui, Nano Lett., 2015, 15, 365.

11 M. Park, J. Im, M. Shin, Y. Min, J. Park, H. Cho, S. Park, M.-B. Shim, S. Jeon, D.-Y. Chung, J. Bae, J. Park, U. Jeong and K. Kim, Nat. Nanotechnol., 2012, 7, 803.

12 K. Yamashita, Y. Yoshioka, K. Higashisaka, Y. Morishita, T. Yoshida, M. Fujimura, H. Kayamuro, H. Nabeshi, T. Yamashita, K. Nagano, Y. Abe, H. Kamada, Y. Kawai, T. Mayumi, T. Yoshikawa, N. Itoh, S. Tsunoda and Y. Tsutsumi, Inflammation, 2010, 33, 276.

13 S. Sharifi, S. Behzadi, S. Laurent, M. L. Forrest, P. Stroeve and M. Mahmoudi, Chem. Soc. Rev., 2012, 41, 2323.

14 S. Maity, A. Chatterjee, B. Singh and A. P. Singh, J. Text. Inst., 2014, 105, 887.
15 A. Laforgue, J. Mater. Chem., 2010, 20, 8233.

16 S. Ji, W. He, K. Wang, Y. Ran and C. Ye, Small, 2014, 10, 4951.

17 O. Yarimaga, B. Yoon, D. Y. Ham, J. Lee, M. Hara, Y. K. Choi and J. M. Kim, J. Mater. Chem., 2011, 21, 18605.

18 M. V. Fabretto, D. R. Evans, M. Mueller, K. Zuber, P. HojatiTalemi, R. D. Short, G. G. Wallace and P. J. Murphy, Chem. Mater., 2012, 24, 3998.

19 M. Yazdimamaghani, M. Razavi, M. Mozafari, D. Vashaee, H. Kotturi and L. Tayebi, J. Mater. Sci.: Mater. Med., 2015, 26, 274 .

20 L. Jin, T. Wang, Z. Q. Feng, M. K. Leach, J. H. Wu, S. J. Mo and Q. Jiang, J. Mater. Chem. B, 2013, 1, 1818.

21 S. C. Luo, E. M. Ali, N. C. Tansil, H. H. Yu, S. Gao, E. A. B. Kantchev and J. Y. Ying, Langmuir, 2008, 24, 8071.

22 H. Okuzaki, H. Suzuki and T. Ito, J. Phys. Chem. B, 2009, 113, 11378.

23 B.-U. Hwang, J.-H. Lee, T. Tran Quang, E. Roh, D.-I. Kim, S.-W. Kim and N.-E. Lee, ACS Nano, 2015, 9, 8801.

24 P. Li, K. Sun and J. Ouyang, ACS Appl. Mater. Interfaces, 2015, 7, 18415.

25 A. A. Balandin, S. Ghosh, W. Bao, I. Calizo, D. Teweldebrhan, F. Miao and C. N. Lau, Nano Lett., 2008, 8, 902.

26 X. Huang, Z. Zeng, Z. Fan, J. Liu and H. Zhang, Adv. Mater., 2012, 24, 5979.

27 D. H. Du, P. C. Li and J. Ouyang, ACS Appl. Mater. Interfaces, 2015, 7, 26952.

28 D. H. Du, P. C. Li and J. Ouyang, J. Mater. Chem. C, 2016, 4, 3224.

29 D. H. Du, H. O. Song, Y. T. Nie, X. H. Sun, L. Chen and J. Y. Ouyang, J. Phys. Chem. C, 2015, 119, 20085.

30 T. Rath and P. P. Kundu, RSC Adv., 2015, 5, 26666.

31 S. F. Pei, J. P. Zhao, J. H. Du, W. C. Ren and H. M. Cheng, Carbon, 2010, 48, 4466.

32 D. Guo, P. Cai, J. Sun, W. He, X. Wu, T. Zhang, X. Wang and X. Zhang, Carbon, 2016, 99, 571.

33 S. Stankovich, D. A. Dikin, R. D. Piner, K. A. Kohlhaas, A. Kleinhammes, Y. Jia, Y. Wu, S. T. Nguyen and R. S. Ruoff, Carbon, 2007, 45, 1558.

34 K. Dagci and M. Alanyalioglu, ACS Appl. Mater. Interfaces, 2016, 8, 2713.

35 P. C. Li, D. H. Du, L. Guo, Y. X. Guo and J. Ouyang, J. Mater. Chem. C, 2016, 4, 6525.

36 D. A. Mengistie, M. A. Ibrahem, P. C. Wang and C. W. Chu, ACS Appl. Mater. Interfaces, 2014, 6, 2292.

37 J. Ouyang, Displays, 2013, 34, 423.

38 L. Zhang, H. Deng, S. Liu, Q. Zhang, F. Chen and Q. Fu, RSC Adv., 2015, 5, 105592.

39 Y. Xia and J. Ouyang, ACS Appl. Mater. Interfaces, 2010, 2, 474.

40 Y. J. Xia, K. Sun and J. Ouyang, Adv. Mater., 2012, 24, 2436.

41 C. Lee, X. D. Wei, J. W. Kysar and J. Hone, Science, 2008, 321, 385.

42 M. D. Stoller, S. Park, Y. Zhu, J. An and R. S. Ruoff, Nano Lett., 2008, 8, 3498.

43 L. J. Zhou, L. Q. Yu, M. M. Ding, J. H. Li, H. Tan, Z. G. Wang and Q. A. Fu, Macromolecules, 2011, 44, 857. 